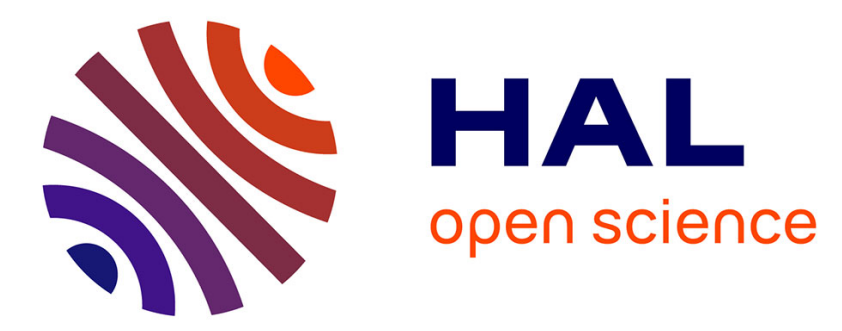

\title{
Improved ion acceleration via laser surface plasma waves excitation
}

\author{
Alessandra Bigongiari, Michèle Raynaud, Caterina Riconda, Anne Héron
}

\section{To cite this version:}

Alessandra Bigongiari, Michèle Raynaud, Caterina Riconda, Anne Héron. Improved ion acceleration via laser surface plasma waves excitation. Physics of Plasmas, 2013, 20 (5), pp.052701. 10.1063/1.4802989 . hal-01327410

\section{HAL Id: hal-01327410 https://hal.science/hal-01327410}

Submitted on 8 Jun 2016

HAL is a multi-disciplinary open access archive for the deposit and dissemination of scientific research documents, whether they are published or not. The documents may come from teaching and research institutions in France or abroad, or from public or private research centers.
L'archive ouverte pluridisciplinaire HAL, est destinée au dépôt et à la diffusion de documents scientifiques de niveau recherche, publiés ou non, émanant des établissements d'enseignement et de recherche français ou étrangers, des laboratoires publics ou privés. 


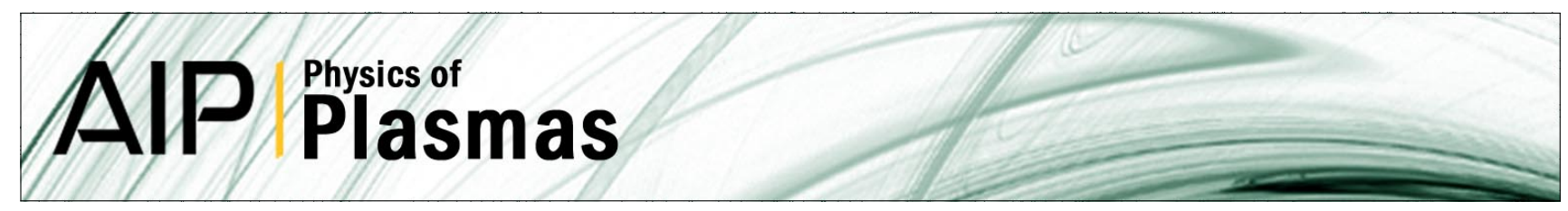

\section{Improved ion acceleration via laser surface plasma waves excitation}

A. Bigongiari, M. Raynaud, C. Riconda, and A. Héron

Citation: Phys. Plasmas 20, 052701 (2013); doi: 10.1063/1.4802989

View online: http://dx.doi.org/10.1063/1.4802989

View Table of Contents: http://pop.aip.org/resource/1/PHPAEN/v20/i5

Published by the American Institute of Physics.

\section{Additional information on Phys. Plasmas}

Journal Homepage: http://pop.aip.org/

Journal Information: http://pop.aip.org/about/about_the_journal

Top downloads: http://pop.aip.org/features/most_downloaded

Information for Authors: http://pop.aip.org/authors

\section{ADVERTISEMENT}

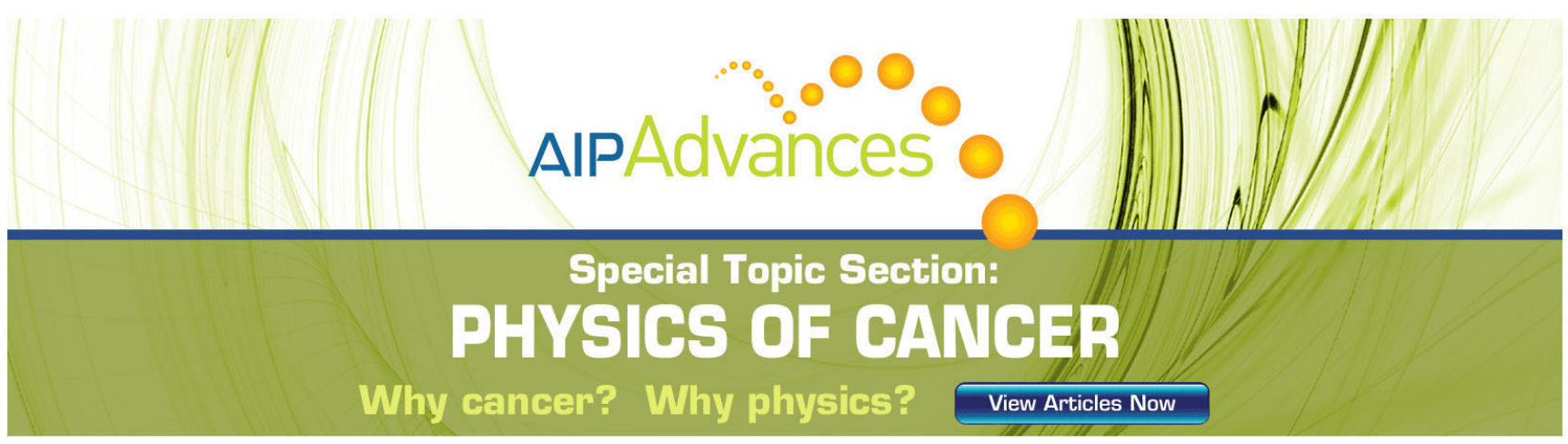




\title{
Improved ion acceleration via laser surface plasma waves excitation
}

\author{
A. Bigongiari, ${ }^{1,2}$ M. Raynaud, ${ }^{1}$ C. Riconda ${ }^{2}$ and A. Héron ${ }^{3}$ \\ ${ }^{1}$ CEA/DSM/LSI, CNRS, Ecole Polytechnique, 91128 Palaiseau Cedex, France \\ ${ }^{2}$ TIPS/LULI, Université Paris 6, CNRS, CEA, Ecole Polytechnique, 3, rue Galilée, \\ 94200 Ivry-sur-Seine, France \\ ${ }^{3}$ CPHT, CNRS, Ecole Polytechnique, 91128 Palaiseau Cedex, France
}

(Received 28 January 2013; accepted 12 April 2013; published online 3 May 2013)

The possibility of enhancing the emission of the ions accelerated in the interaction of a high intensity ultra-short $(<100 \mathrm{fs})$ laser pulse with a thin target $\left(<10 \lambda_{0}\right)$, via surface plasma wave excitation is investigated. Two-dimensional particle-in-cell simulations are performed for laser intensities ranging from $10^{19}$ to $10^{20} \mathrm{Wcm}^{-2} \mu \mathrm{m}^{2}$. The surface wave is resonantly excited by the laser via the coupling with a modulation at the target surface. In the cases where the surface wave is excited, we find an enhancement of the maximum ion energy of a factor $\sim 2$ compared to the cases where the target surface is flat. (C) 2013 AIP Publishing LLC. [http://dx.doi.org/10.1063/1.4802989]

\section{INTRODUCTION}

During the past decade, with the rapid development of the laser technology, ion acceleration has received extensive attention in the area of laser-plasma interaction. This has been motivated by the discovery of multi-MeV protons in three experiments performed independently in the year $2000,{ }^{1-3}$ where an ultra intense laser pulse $I \lambda_{0}^{2}>10^{18} \mathrm{Wcm}^{-2} \mu \mathrm{m}^{2}$ was impinging on metallic targets. The protons are present as contaminants on the target surface and are accelerated by the sheath field generated near the target surface due to charge separation, according to the Target Normal Sheat Acceleration (TNSA) model. ${ }^{4,5}$ The characteristics of the ion emission at the rear (unirradiated) side of the target attracted more attention due to the high degree of collimation, which is suitable for applications requiring highly localized energy deposition in dense matter, such as proton therapy ${ }^{6-9}$ and laser fusion. ${ }^{10}$ However, the energy required for such applications is $>65 \mathrm{MeV}$ (the latter is the approximative value required for the treatment of eye tumours by proton therapy), while the energy that is obtained in experiments is just few tens of MeV. ${ }^{8}$ For example, in Refs. 2 and 3, proton beams up to $45 \mathrm{MeV}$ were measured focusing a laser beam of $100 \mathrm{~J}$ onto thin foil targets of few tens microns thickness, with a duration in the ps range while in Ref. $910 \mathrm{MeV}$ protons are obtained using a laser beam of $0.6 \mathrm{~J}$ having duration $30 \mathrm{fs}$. Longer pulses can deliver higher energies, accelerating a larger number of protons with a greater cut off energy compared to shorter pulses. However, for applications, the repetition rate has to be considered, which is higher for shorter pulses: a pulse in the ps range has a typical repetition rate of one shot every $20 \mathrm{~min}$ while for the 30 fs pulse in Ref. 9, the repetition rate was $10 \mathrm{~Hz}$. For this reason, we are interested in the possibility of increasing the proton energy in the regime of the short laser pulses, enhancing the conversion of the laser energy into energetic proton beams.

We present here a numerical study of the enhanced proton acceleration using structured targets which in particular allow the excitation of surface plasma waves (SPWs). ${ }^{11-16}$ Surface waves are supported by a stepwise profile, propagate along the plasma-vacuum interface and are characterized by a localized, high frequency, resonant electric field. In a precedent work, ${ }^{17}$ we have shown the possibility of resonant excitation of SPW by ultrashort high intensity laser pulse $\left(\left[10^{15}-10^{20}\right] \mathrm{Wcm}^{-2} \mu \mathrm{m}^{2}\right)$ in an overdense $\left(\omega_{p e}>\omega_{0}\right.$, with $\omega_{p e}$ the plasma frequency, $\omega_{0}$ the laser frequency and $n_{e}=25-100 n_{c}$, with $n_{e}$ the electron density and $n_{c}$ the critical density) pre-structured plasma. A dramatic increase of both the laser absorption (up to 80\%) and the electron energy was observed, which can be associated with the characteristics of the resonant field.

In this paper, we wish to investigate in detail the process of ion acceleration for high intensity short laser pulses ( $\left[10^{19}-10^{20}\right] \mathrm{Wcm}^{-2} \mu \mathrm{m}^{2}$ ), which correspond to the most common intensity regime for proton acceleration experiments. As the dependence on laser intensity and the target thickness was found to play an important role on the maximum ion energy, ${ }^{18-21}$ we will analyze here the influence of these two parameters in the presence of surface wave excitation.

\section{PIC SIMULATIONS}

Two-dimensional Particle-In-Cell (PIC), simulations were performed by using the code EMI2D. ${ }^{22}$ An overdense plasma with $n_{e}=25 n_{c}$ is considered here with a steep density gradient along the $x$ direction of the $(x, y)$ simulation plane. The plasma is initially described by a Maxwellian distribution with an electron temperature $T_{e}=1 \mathrm{keV}$ (thermal velocity $v_{t h}=0.044 c$ ) and an ion temperature $T_{i}=T_{e} / 10$ (here, $m_{i} / m_{e}=1836$, where $m_{i}$ and $m_{e}$ are the ion and electron mass). The charge of the ions was $Z=1$. The grid size was $\triangle x \approx 5 \lambda_{D}$ and $\triangle y \approx 4 \lambda_{D}$ (therefore, it depends on the initial plasma density taken in the simulation), where $\lambda_{D}=v_{t h} / \omega_{p e}$ is the Debye length and the number of particles per cell was $\mathrm{NP}=100$. The incoming laser beam is $P$-polarized as it is the only possible polarization for the surface plasma wave excitation so that the laser electric field is in the $(x, y)$ simulation plane. The laser has angular frequency $\omega_{0}$ and wavelength $\lambda_{0}$ and is characterized by a temporal Gaussian shape corresponding to a pulse duration of $\tau_{L}=240 \omega_{0}^{-1}$ (FWHM, $\simeq 127\left(\frac{\lambda_{0}}{\mu \mathrm{m}}\right) \mathrm{fs}$ ). In a first set of simulations (Secs. III and IV), we consider a uniform pulse profile along $y$. In Sec. V, we 
will consider the case of a Gaussian transverse profile and show that the SPW induced enhancement of the ion energy is conserved. Different laser intensities have been studied from $10^{19}$ to $10^{20} \mathrm{Wcm}^{-2} \mu \mathrm{m}^{2}$.

As it is well known the surface wave can be associated with an electric field of the form: $E_{S P W}(x) e^{i(\omega t-k y)}$, with $E_{S P W}(x)=E_{S P W}(0) \times\left[\Theta(-x) \exp \left(x / L_{E}\right)+\Theta(x) \exp \left(-x / L_{E p l}\right)\right]$. Here, $\Theta(x)$ denotes the Heaviside step function, $x=0$ corresponds to the position of the plasma-vacuum interface, and $L_{E}$ and $L_{E p l}$ are the evanescence lengths of the surface wave in vacuum and inside the plasma, respectively, given by $L_{E}=1 / \sqrt{k^{2}-\omega^{2} / c^{2}}$ and $L_{E p l}=L_{E} /\left(\omega_{p e}^{2} / \omega^{2}-1\right)$. The full form of the field can be found in Ref. 23. As the phase velocity of a surface wave is lower than the light velocity, to satisfy the phase matching conditions we have considered a plasma surface having a periodic shape along the $y$ direction such that $k=k_{0}\left(\sin \theta+\lambda_{0} / a_{m}\right)$, where $k_{0}=\omega_{0} / c$ is the laser field wave vector, $a_{m}$ denotes the surface density period, and $\theta$ is the laser incidence angle. The dispersion relation of the surface plasma wave in the limit of low temperature $v_{t h} / c \ll 1$, as it is our case at the beginning of the interaction, takes the form $k^{2} c^{2} / \omega^{2}=\left(\omega^{2}-\omega_{p e}^{2}\right) /\left(2 \omega^{2}-\omega_{p e}^{2}\right)$. The phase matching conditions combined with the surface wave dispersion relation lead to the resonance condition, in terms of plasma and laser parameters, $\sin (\theta)+\lambda_{0} / a_{m}$ $=\sqrt{\left(1-n_{e} / n_{c}\right) /\left(2-n_{e} / n_{c}\right)} \simeq 1$, where the last equality is valid for $n_{e} / n_{c} \gg 1$. In the following, we have taken an incidence angle of $\theta=30^{\circ}$ with a modulation periodicity of $2 \lambda_{0}$ so that the surface wave dispersion relation is satisfied. The modulation depth, $d$, was equal to $\lambda_{0}$ (from the dip to the tip). In the simulations, the box size is $6 \lambda_{0}$ along the $y$ direction and $76 \lambda_{0}$ along the $x$ direction and there is a vacuum length of $38 \lambda_{0}$ in front of the target, which allows spatial expansion of particles and minimizes edge effects. The thickness of the targets considered varies between $h=3.5 \lambda_{0}$ and $8 \lambda_{0}$. In order to compare the effects of the SPW excitation with a standard target, each simulation was complemented with simulations having the same characteristics but a flat plasma-vacuum interface.

\section{ELECTRON HEATING IN PRESENCE OF A SPW}

In high intensity short pulse laser-overdense plasma interaction, the electromagnetic energy is transferred to the plasma via electron heating, which occurs during the interaction due to different collisionless mechanisms, ${ }^{24}$ such as sheath inverse bremsstrahlung, ${ }^{25} \vec{J} \times \vec{B}$ heating, ${ }^{26}$ vacuum heating, ${ }^{27}$ and anomalous skin-layer heating. ${ }^{28}$ In particular, very energetic electrons are generated, ${ }^{29}$ which are referred as hot electrons, i.e., electron having energy much higher than the thermal energy. Ions are then accelerated by the sheath field, which is set up by the hot electrons that are expanding towards the vacuum, according to the TNSA model. ${ }^{4,5}$ In presence of a surface wave excited at the vacuum-plasma interface, the mechanism of electron heating changes, as discussed in Ref. 17, as the component of the electric field perpendicular to the surface is amplified and the field is more localized. Thus, in order to describe the effects of SPW excitation on the acceleration of ions,

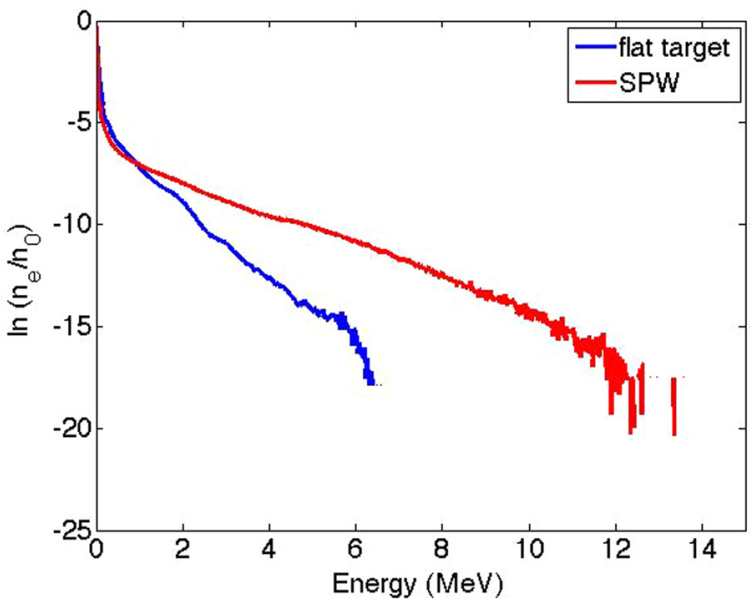

FIG. 1. Electron energy spectra for the flat target (in blue) (no SPW excitation) and the grating target (in red) case for $I \lambda_{0}^{2}=10^{19} \mathrm{Wcm}^{-2} \mu \mathrm{m}^{2}$, at $t=500 \omega_{0}{ }^{-1}$. The target thickness is $h=3.5 \lambda_{0} . n_{0}$ is the total electron density integrated over the energy.

we have to consider first the characteristics of electron heating. We start presenting the electron energy distribution function for a reference case: a laminar target having thickness $h=3.5 \lambda_{0}$, at the laser intensity $I \lambda_{0}^{2}=10^{19} \mathrm{Wcm}^{-2} \mu \mathrm{m}^{2}$. As we will see in the following, this case represents the best setup for the enhancement of the electron heating via SPW excitation in our configuration. The electron energy spectra are shown in Fig. 1 for the case where the SPW is excited and for the flat target, at the time $t=500 \omega_{0}{ }^{-1}$, which corresponds approximatively to the time at which the SPW field reaches its maximum amplitude.

As a consequence of SPW excitation, we observe an enhancement of the electron heating: the number and energy of the hot electron is increased compared to the case of a flat target where no SPW is excited. In Fig. 1, we observe two different populations of electrons: "cold" electrons, which have gained less energy during the interaction and "hot" electrons, which have been efficiently heated and have gained a considerable amount of energy. Part of the energy absorbed from the laser when the SPW is excited is converted to "hot" electrons flowing parallel to the front surface of the plasma ${ }^{17}$ and does not participate to the bulk heating. However, the majority of the "hot" electrons crosses the target toward the rear surface. The two populations, "hot" and "cold" electrons, are associated to two quasi-exponential slopes observed in the spectra, in the low and high energy range. Thus, assuming ${ }^{4,30}$ that the electrons have a distribution function of the form $n_{e}(E) \sim \exp \left(-E / k_{B} T_{e}\right)$, we can derive the electron temperature for the two populations and define $T_{\text {cold }}$ and $T_{\text {hot }}$. In the following, we will use these two parameters as well as the maximum energy $E_{\text {max }}$ to describe the electron spectra.

Comparing the hot electron temperatures in Table I, we find that in the case where the surface wave is excited $T_{\text {hot }}$ and the maximum electron energy $E_{\max }$ are increased up to a factor $\sim 4$ and $\sim 2$, respectively. For example, for $I \lambda_{0}^{2}=10^{19} \mathrm{Wcm}^{-2} \mu \mathrm{m}^{2}, T_{\text {hot }}$ increases from $600 \mathrm{MeV}$ to $2.3 \mathrm{MeV}$ and $E_{\max }$ increases from $7 \mathrm{MeV}$ to $14 \mathrm{MeV}$. Moreover, the percentage of electrons heated the high energy 
TABLE I. Electron energy and percentage of electrons having energy $>1 \mathrm{MeV}$ as a function of the laser intensity, at $t=500 \omega_{0}{ }^{-1}$ for $h=3.5 \lambda_{0}$ and $h=8 \lambda_{0}$. Two targets type are considered: one having modulated surface such to resonantly excite the SPW (res) and one having flat surface (flat). $a_{0}=\frac{e E_{0}}{m_{e} c \omega}$.

\begin{tabular}{lccccccc}
\hline \hline $\begin{array}{l}\text { Target } \\
\text { type }\end{array}$ & $\begin{array}{c}h \\
\lambda_{0}\end{array}$ & $\begin{array}{c}I \lambda_{0}^{2} \\
\mathrm{Wcm}^{-2} \mu \mathrm{m}^{2}\end{array}$ & $\begin{array}{c}a_{0} \\
\ldots\end{array}$ & $\begin{array}{c}T_{\text {cold }} \\
\mathrm{keV}\end{array}$ & $\begin{array}{c}T_{\text {hot }} \\
\mathrm{MeV}\end{array}$ & $\begin{array}{c}E_{\text {max }} \\
\mathrm{MeV}\end{array}$ & $\begin{array}{c}\% \text { hot } \\
n_{\text {hot }} / n_{\text {tot }}\end{array}$ \\
\hline res & 3.5 & $10^{19}$ & 2.72 & 54 & 2.3 & 14 & $5 \%$ \\
flat & 3.5 & $10^{19}$ & 2.72 & 38 & 0.6 & 7 & $1 \%$ \\
res & 8 & $10^{19}$ & 2.72 & 54 & 2.1 & 13.5 & $3 \%$ \\
flat & 8 & $10^{19}$ & 2.72 & 36 & 0.6 & 6 & $0.6 \%$ \\
res & 3.5 & $5 \times 10^{19}$ & 6.1 & 350 & 3.5 & 48 & $23 \%$ \\
flat & 3.5 & $5 \times 10^{19}$ & 6.1 & 320 & 1.6 & 27 & $12 \%$ \\
res & 3.5 & $10^{20}$ & 8.6 & 500 & 4.7 & 52 & $38 \%$ \\
flat & 3.5 & $10^{20}$ & 8.6 & 500 & 2.9 & 45 & $11 \%$ \\
res & 8 & $10^{20}$ & 8.6 & 450 & 3.9 & 52 & $17 \%$ \\
flat & 8 & $10^{20}$ & 8.6 & 450 & 2.9 & 44 & $11 \%$ \\
\hline \hline
\end{tabular}

range is increased: for $I \lambda_{0}^{2}=10^{19} \mathrm{Wcm}^{-2} \mu \mathrm{m}^{2}$ and $h=3.5 \lambda_{0}$, the electrons having energy greater than $1 \mathrm{MeV}$ are $5 \%$ of the total when the SPW is excited and $1 \%$ for the flat target.

For the highest laser intensity value $\left(I \lambda_{0}^{2}=10^{20}\right.$ $\mathrm{Wcm}{ }^{-2} \mu \mathrm{m}^{2}$ ), the hot electron temperature and maximum energy observed when the SPW is present tend to saturate and become closer to the values for the flat target: for $h=3.5 \lambda_{0}, E_{\max }$ only increases from $45 \mathrm{MeV}$ to $52 \mathrm{MeV}$ when the surface wave is excited and $T_{h o t}$ increases of a factor $<2$. Nevertheless, the percentage of electrons having energies greater than $1 \mathrm{MeV}$ is still significantly increased when the surface wave is excited, as observed for the lower intensities. We interpret the lessening of the increase in hot electron temperature and maximum energy at the highest intensity as related to the fact that we are approaching the limit of the ultrarelativistic regime $\left(a_{0} \gg 1\right)$, where the ultrarelativistic similarity theory may apply, ${ }^{31}$ inducing a change of regime at the considered density. Moreover, relativistic detuning or plasma expansion (discussed in Sec. IV) reducing the local density can affect the resonance condition. However, all these effects should be considerably limited at higher densities. ${ }^{17}$

For thin laminar targets, the number and temperature of the hot electrons depend also on the target thickness. The hot electrons can recirculate inside the target: ${ }^{18-21}$ electrons accelerated inside the plasma reach the rear (unirradiated) side of the target, where a Debye sheath has formed that reflects them back towards the front (irradiated). If we reduce the target thickness, a larger number of electrons will have the possibility to interact with the driving field at the irradiated side and further increase their energy. For our reference case of thickness, $3.5 \lambda_{0}$ relativistic electron will do a full tour and go back to the irradiated surface in roughly $25 f s$, thus with our choice of pulse duration they can see the laser and, in the case when it is excited, the SPW field more than once. As we will show, although this effect is present also in the case of the flat target, it is considerably more efficient when the SPW is excited.

In Fig. 2, we can observe the spectra corresponding to three target thickness values, for $I \lambda_{0}^{2}=10^{19} \mathrm{Wcm}^{-2} \mu \mathrm{m}^{2}$ :

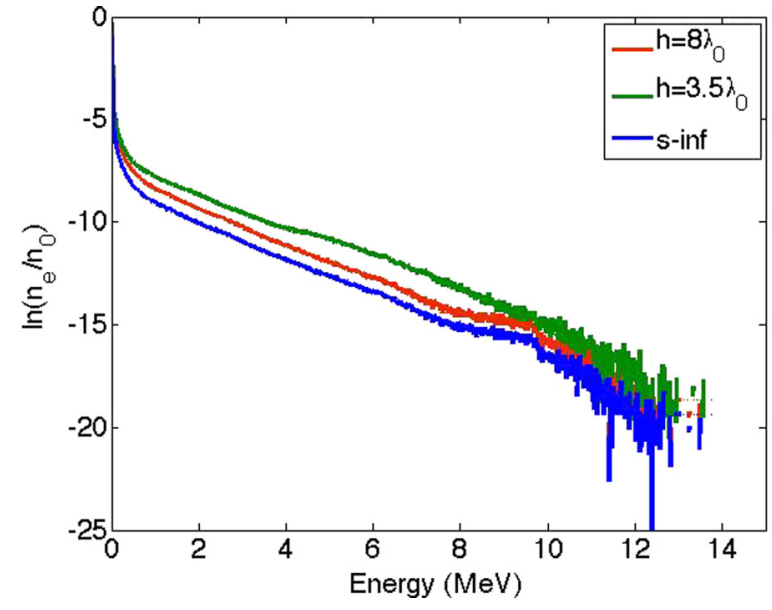

FIG. 2. Electron spectra for the laminar targets having different thickness, $h=3.5 \lambda_{0}, 8 \lambda_{0}$ and the semi-infinite target, taken at time $t=500 \omega_{0}^{-1}$ for $I \lambda_{0}^{2}=10^{19} \mathrm{Wcm}^{-2} \mu \mathrm{m}^{2}\left(a_{0}=2.72\right), n_{e}=25 n_{c}, d=\lambda_{0}$, when the SPW is excited. $n_{0}$ is the total electron density integrated over the energy.

$3.5 \lambda_{0}, 8 \lambda_{0}$ and the limit case of a semi-infinite target. The latter is reproduced performing a simulation where the electrons reaching the right side of the target are cooled in a buffer and re-enter the plasma at the thermal speed, thus they can not recirculate. We observe that the number of electrons that are accelerated in the high-energy range decreases as we increase the target thickness and the worst case is that of the semi-infinite target. Also the hot electron temperature decreases: we have $T_{\text {hot,s-inf }}=1.9 \mathrm{MeV}$ for the semi-infinite case and $T_{h o t}, 8 \lambda_{0}=2.1 \mathrm{MeV}$. We notice that also at $I \lambda_{0}^{2}=10^{20} \mathrm{Wcm}^{-2} \mu \mathrm{m}^{2}$, the production of hot electrons is increased when the target thickness decreases despite the fact that the maximum electron energy $E_{\max }$ does not change. Thus, we may infer that the recirculation tends to raise the number of hot electrons more than their maximum energy.

As hot electron temperature and density are the parameters which determine the TNSA field, ${ }^{4,5}$ their enhancement is of great importance in the process of ion acceleration, as we will discuss in Sec. IV.

\section{ION ACCELERATION}

In the following, we focus both for laminar and grating targets on the acceleration of ions at the unirradiated side, in the direction perpendicular to the surface. If we assume that the ion acceleration at the rear side is driven by the hot electron population, we can estimate the characteristic time $\tau_{h}$ at which the electrons transfer their energy to the ions. ${ }^{32}$ The latter is given by $\tau_{h}=\frac{h}{\sqrt{4 T_{h o t} / m_{i}}}$, which is $\simeq 230 \omega_{0}^{-1}$ for the case having $h=3.5 \lambda_{0}$ (respectively, $\simeq 520 \omega_{0}^{-1}$ for $\left.h=8 \lambda_{0}\right)$ at $I \lambda_{0}^{2}=10^{19} \mathrm{Wcm}^{-2} \mu \mathrm{m}^{2}$. Thus, we will report the ion energies, in Table II, for $t=1000 \omega_{0}^{-1}$, which is at least one $\tau_{h}$ after the time at which the laser intensity reaches its maximum on the target.

When the surface wave is excited the energy of the ions is significantly increased: for example, at $I \lambda_{0}^{2}=10^{19}$ $\mathrm{Wcm}^{-2} \mu \mathrm{m}^{2}$, the cut-off energy of the ions increases from 
TABLE II. Cut-off ion energy as a function of the laser intensity at $t=1000 \omega_{0}{ }^{-1}$ for $h=3.5 \lambda_{0}$ and $h=8 \lambda_{0}$. Two targets type are considered: one having modulated surface such to resonantly excite the SPW (res) and one having flat surface (flat). $a_{0}=\frac{e E_{0}}{m_{e} c \omega}$.

\begin{tabular}{lccccc}
\hline \hline $\begin{array}{l}\text { Target } \\
\text { type }\end{array}$ & $\begin{array}{c}I \lambda_{0}^{2} \\
\mathrm{Wcm}^{-2} \mu \mathrm{m}^{2}\end{array}$ & $\begin{array}{c}a_{0} \\
-\end{array}$ & $\begin{array}{c}h \\
\lambda_{0}\end{array}$ & $\begin{array}{c}E_{\text {ion }} \\
\mathrm{MeV}\end{array}$ & $\begin{array}{c}\%>\mathrm{MeV} \\
n_{i,>\mathrm{MeV}} / n_{i, \text { rear }}\end{array}$ \\
\hline res & $10^{19}$ & 2.72 & 3.5 & 14 & $40 \%$ \\
flat & $10^{19}$ & 2.72 & 3.5 & 8 & $18 \%$ \\
res & $10^{19}$ & 2.72 & 8 & 11 & $36 \%$ \\
flat & $10^{19}$ & 2.72 & 8 & 6.1 & $18 \%$ \\
res & $5 \times 10^{19}$ & 6.1 & 3.5 & 35 & $62 \%$ \\
flat & $5 \times 10^{19}$ & 6.1 & 3.5 & 25 & $44 \%$ \\
res & $10^{20}$ & 8.6 & 3.5 & 50 & $67 \%$ \\
flat & $10^{20}$ & 8.6 & 3.5 & 42 & $60 \%$ \\
res & $10^{20}$ & 8.6 & 8 & 42 & $80 \%$ \\
flat & $10^{20}$ & 8.6 & 8 & 37 & $59 \%$ \\
\hline \hline
\end{tabular}

$6 \mathrm{MeV}$ to $11 \mathrm{MeV}$ for a target thickness of $8 \lambda_{0}$ and from $8 \mathrm{MeV}$ to $14 \mathrm{MeV}$ for a target thickness of $3.5 \lambda_{0}$. For the highest laser intensity and $h=3.5 \lambda_{0}$ value, the maximum ion energy is $50 \mathrm{MeV}$ at $t=1000 \omega_{0}^{-1}$. The number of ions accelerated in the high energy range is also increased as shown in Fig. 3, which reproduces the ion density distribution as a function of energy at $I \lambda_{0}^{2}=10^{19} \mathrm{Wcm}^{-2} \mu \mathrm{m}^{2}$ for the flat target and when the SPW is excited, for $h=3.5 \lambda_{0}$. In the last column of Table II are given, for each case, the percentage of ions accelerated at the unirradiated target having energy greater than $1 \mathrm{MeV}$, calculated considering the ion phase space extending from the position of the rear surface $x_{\text {rear }}$ to the right boundary of the simulation box. The latter is given with the purpose of comparing the ion energy distributions of the flat and grating targets for a given laser intensity and target thickness. When the surface wave is excited, the percentage of ions in the $\mathrm{MeV}$ range increases from $18 \%$ to $40 \%$ of the total number of ions accelerated at the rear surface. We

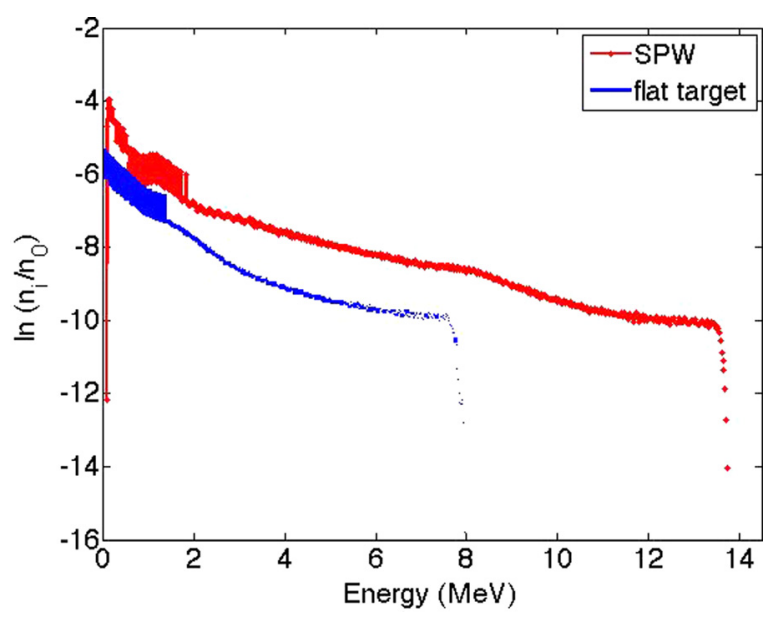

FIG. 3. Density distribution of the ions accelerated at the rear surface (obtained considering the ions in the space between the rear target surface at $x=262 k_{0}^{-1}$ and the right edge of the simulation box, at $x=480 k_{0}^{-1}$ ) as a function of energy, for the flat target and when the SPW is excited at time $t=1000 \omega_{0}^{-1} \cdot h=3.5 \lambda_{0}, I \lambda_{0}^{2}=10^{19} \mathrm{Wcm}^{-2} \mu \mathrm{m}^{2}\left(a_{0}=2.72\right), n_{e}=25 n_{c}$, $d=\lambda_{0} . n_{0}$ is the ion density integrated over the energy. observe that the ion energy is higher for the thinner target, both for the grating and flat target and that, when the surface wave is excited the maximum ion energy is increased up to a factor $\xi \simeq 1.8$. This enhancement factor is close to the maximum enhancement that was found for the electron energy, when a surface wave is excited compared to the flat target, consistent with the idea that the ion energy increases as an effect of the enhancement of the TNSA field. The reduction of the ion energy with the target thickness, instead, can be interpreted as an effect of hot electrons recirculation, ${ }^{18-21}$ which gives a contribution to the TNSA field, increasing the density of hot electrons at the surface.

For $I \lambda_{0}^{2}=10^{20} \mathrm{Wcm}^{-2} \mu \mathrm{m}^{2}$, the ion energy for the flat target becomes closer to the values observed when the SPW is present, coherently with the hot electron parameters found at this laser intensity, which were discussed in Sec. III. However, the hot electrons are not the only population to consider in the generation of the TNSA field: also the cold electrons play an important role as their temperature determines the expansion of the bulk plasma. ${ }^{32}$ The time scale for the expansion of a plasma foil of thickness $h$ is given by the "disassembly time" $t_{d}=\frac{h}{2 c_{s}}, 5,32$ where $c_{s}=\sqrt{T_{\text {cold }} / m_{i}}$, which is $\simeq 1450 \omega_{0}^{-1}$ at $I \lambda_{0}^{2}=10^{19} \mathrm{Wcm}^{-2} \mu \mathrm{m}^{2}$ and $\simeq 450 \omega_{0}^{-1}$ at $I \lambda_{0}^{2}=10^{20} \mathrm{Wcm}^{-2} \mu \mathrm{m}^{2}$, for $h=3.5 \lambda_{0}$. This means that for $I \lambda_{0}^{2}=10^{20} \mathrm{Wcm}^{-2} \mu \mathrm{m}^{2}$, the target expansion is much faster than for $I \lambda_{0}^{2}=10^{19} \mathrm{Wcm}^{-2} \mu \mathrm{m}^{2}$, as it is shown also from the ion density profiles in Fig. 4. The rapid expansion may modify the scale length of the plasma at the unirradiated surface, reducing the strength of the accelerating field.

To summarize, we find a significant enhancement of ion acceleration via the excitation of a surface wave on a grating target and we can determine the optimal laser intensity range for the maximum efficiency of the process.

\section{THE GAUSSIAN PULSE}

In this section, we will examine the effect of the finite transversal size of the laser pulse envelope, by approximating its $y$ profile with a Gaussian function. In this case, we expect that, far from the laser spot, the electron heating gradually loses its efficiency, due to the decrease of the laser

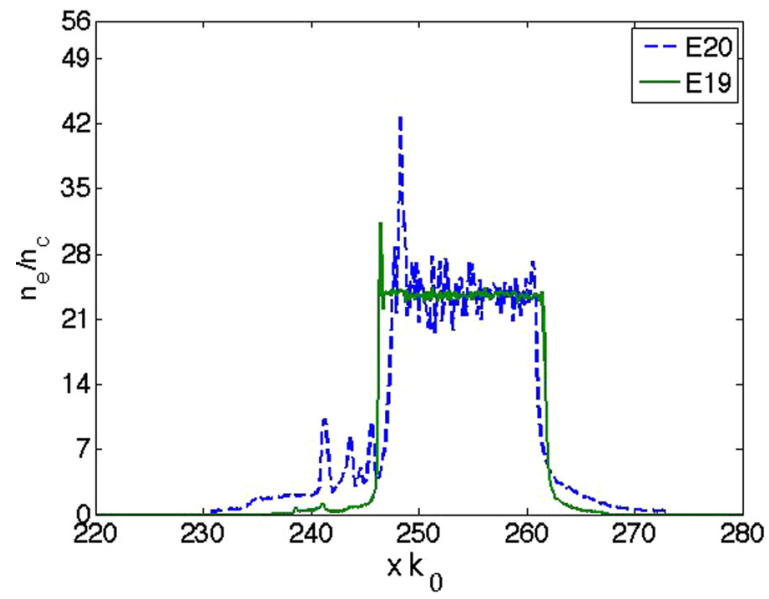

FIG. 4. Density profile of the ions as a function of $x$ at $y=22 k_{0}^{-1}$ for $I \lambda_{0}^{2}=10^{19} \mathrm{Wcm}^{-2} \mu \mathrm{m}^{2}$ and $I \lambda_{0}^{2}=10^{20} \mathrm{Wcm}^{-2} \mu \mathrm{m}^{2}$ at $t=500 \omega_{0}^{-1}$. $h=3.5 \lambda_{0}, n_{e}=25 n_{c}$, and $d=\lambda_{0}$. 
intensity on the target surface. Moreover, for small spot sizes as commonly used, we expect the non homogeneity of the laser intensity along $y$ to affect electron recirculation. Namely, the electrons which are accelerated inside the target in the directions non-perpendicular to the target may not come back at the focal spot after the reflection at the rear side. The pulse will be focused on the center of the target surface, with a focal spot (intended as the FWHM of the Gaussian) of $10 \lambda_{0}$, and will have the same temporal dependence as in the previous sections. This focal spot size corresponds to the typical transverse size of the pulse in the experiments where the interaction of a laser with a laminar target is studied. ${ }^{3,33,34}$ In the simulation, we have used a thickness of $3.5 \lambda_{0}$ and density $n_{e}=25 n_{c}$, with a modulation at the surface of periodicity $a_{m}=2 \lambda_{0}$ and depth $d=\lambda_{0}$. The pulse is incident at the resonance angle $\theta=30^{\circ}$. As the pulse is no more periodic in the $y$ direction, we used a simulation box size of $110 \lambda_{0} \simeq 691 k_{0}^{-1}$ along $y$, in order to avoid unwanted effects from the boundaries (which have periodic conditions along $y$ ), while the total box size will be $480 k_{0}^{-1}$ along $x$.

In order to give an overview of the simulation set-up, in Fig. 5, we show the $x$ component of the electric field, for the Gaussian pulse. If we consider the focal spot area, the surface wave field observed in the simulation is practically identical to the one observed in the case of a uniform laser profile, as we observe in Fig. 6. The basic characteristics of SPW excitation and electron heating (discussed in a previous publication ${ }^{17}$ ) are not modified by the different laser shape if we consider the focal spot area. However, as we expected, the hot electron production is limited to the focal spot area and their temperature is lower: $T_{\text {hot,Gaussian }}=1.4 \mathrm{MeV}$ (instead of $2.3 \mathrm{MeV}$ in the case of a uniform pulse profile) and $E_{\text {max,Gaussian }}=11 \mathrm{MeV}$ (instead of $14 \mathrm{MeV}$ in the case of a uniform pulse profile) at $t=500 \omega_{0}^{-1}$. Therefore, the TNSA field whose strength depends on the electron temperature is lower and the final ion energy is reduced: at $t=1000 \omega_{0}^{-1}$, we have a maximum ion energy of $7.1 \mathrm{MeV}$, i.e., a half of the value obtained for an uniform laser intensity on the surface. The ions are accelerated, both at the rear and at the

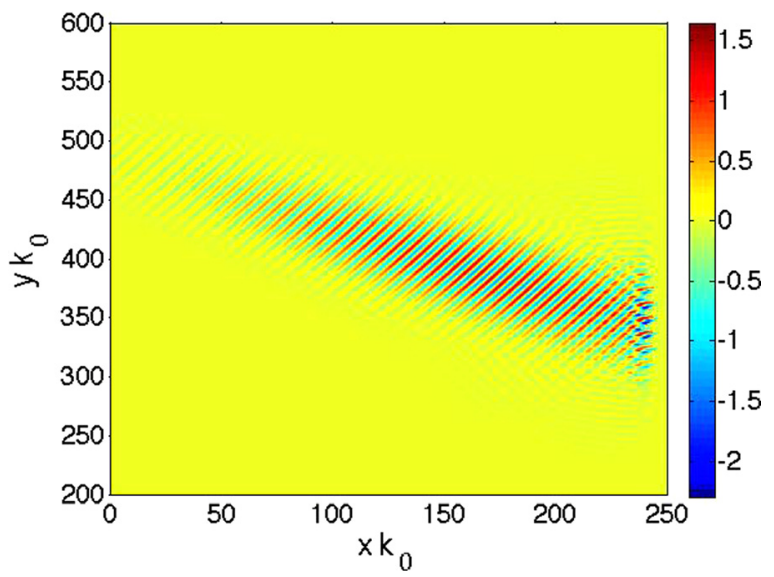

FIG. 5. Electric field $E_{x}$ at $t=390 \omega_{0}^{-1}$ for the Gaussian pulse, $I \lambda_{0}^{2}=10^{19}$ $\mathrm{Wcm}^{-2} \mu \mathrm{m}^{2}\left(a_{0}=2.72\right), d=\lambda_{0}, n_{e}=25 n_{c}$, semi-infinite target when the SPW is excited by a Gaussian laser pulse. The plasma starts at $x=240 k_{0}^{-1}$, the pulse focus is at $y \simeq 345.5 k_{0}^{-1}$, and the focal spot size is $10 \lambda_{0} \simeq 63 k_{0}^{-1}$.
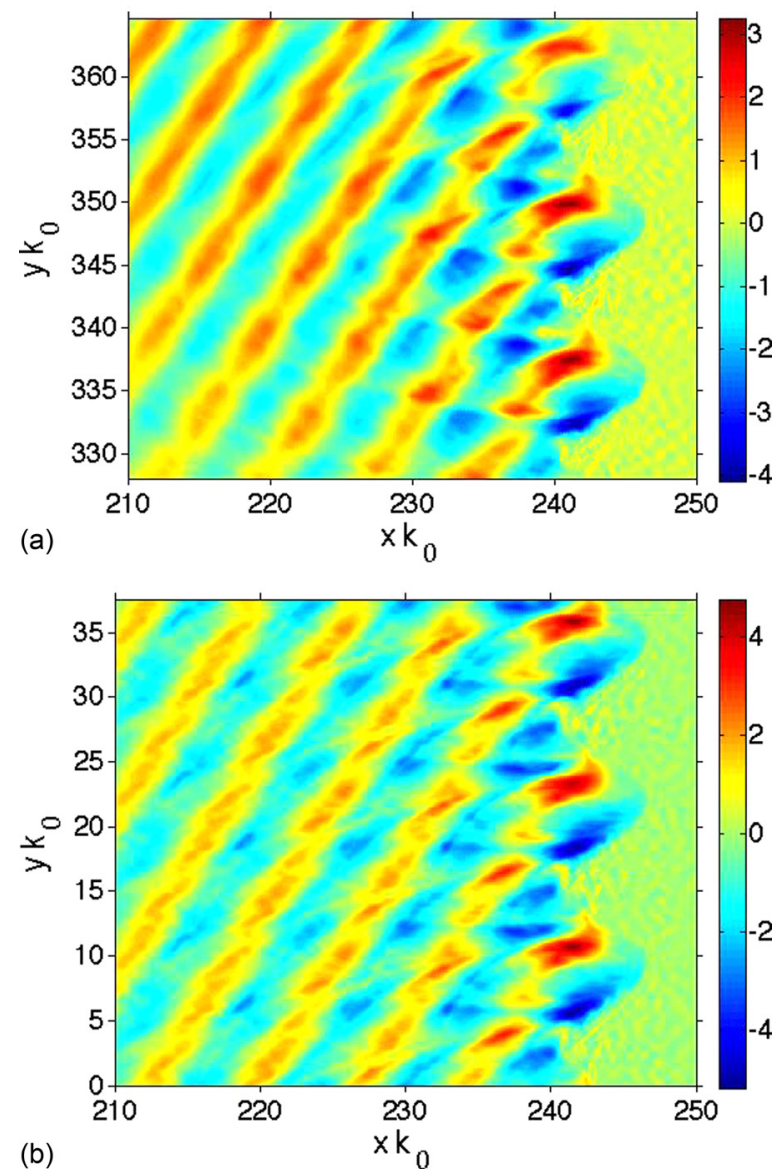

FIG. 6. $x$ component of the SPW and exciting laser field in the $x y$ plane (in each figure, the vacuum is on the left and the plasma on the right, the modulated surface being at $x=240 k_{0}^{-1}$ ), at its maximum amplitude $\left(t_{E \max }=492 \omega_{0}^{-1}\right)$ for the Gaussian pulse shape (left) and the uniform $y$ profile (right), $I \lambda_{0}^{2}=10^{19} \mathrm{Wcm}^{-2} \mu \mathrm{m}^{2}$. The field is in units of $E_{0}$, and the lengths are in units of $k_{0}^{-1}$.

front sides, around the focal spot position in $y$, which corresponds to the maximum hot electron density at the surface. Fig. 7 shows the normalized ion momentum $\left(p_{x} / m_{i} c\right)$ distribution as a function of $y$, for $t=500 \omega_{0}^{-1}$, in which we can

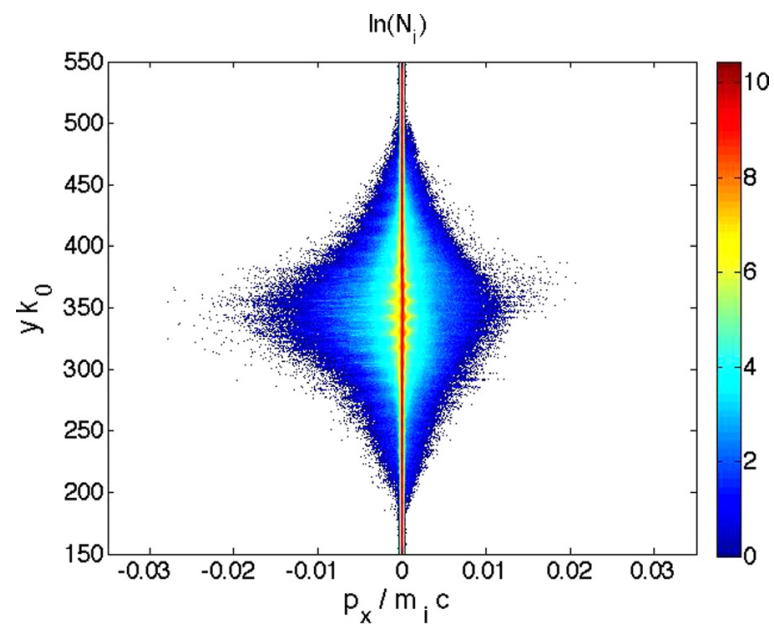

FIG. 7. Ion phase space $\left(y, p_{x}\right)$ for the laminar target of thickness $h=3.5 \lambda_{0}$ when SPW is excited by a Gaussian laser pulse; $I \lambda_{0}^{2}=10^{19} \mathrm{Wcm}^{-2} \mu \mathrm{m}^{2}$ $\left(a_{0}=2.72\right), n_{e}=25 n_{c}, t=500 \omega_{0}^{-1}$, and $d=\lambda_{0} . N_{i}$ is the ion number at a given position. 
observe a peak around $y \simeq 345.5 k_{0}^{-1}$, where the hot electron density is higher. The non homogeneity of the hot electron density at the rear surface tends also to increase the angular spread of the ions about the direction normal to the surface, which rises from $2^{\circ}$ to $7^{\circ}$ compared with the case of uniform laser intensity.

Nevertheless, the same ion energy reduction and angular spread increase is observed also for a plane laminar target when the laser pulse has a Gaussian envelope: if we consider a simulation having the same laser and target parameters as the ones described above, but flat target surface, the maximum energy of the ions emitted at the unirradiated side is $4 \mathrm{MeV}$. This means that, when the SPW is excited, the maximum ion energy is increased of the same factor $\simeq 1.8$ (compared to a plane target) that is found in the case where the laser has uniform intensity in the transverse direction. We may conclude that the finite transverse size is not expected to affect the enhancement factor due to the surface wave field and our simulations predict the possibility to increase the maximum energy of laser accelerated ions of a factor $\simeq 1.8$ in the intensity range that we have analysed. Moreover, as we find that the decrease of the ion energy for the case of the Gaussian pulse is mainly due to the reduction of electron recirculation, we do not expect such a discrepancy for target thickness greater than $\sim \tau_{L} c$.

\section{CONCLUSIONS}

We have studied, via PIC simulation, the effects of SPW excitation on laser accelerated ions for an overdense plasma $\left(n_{e}=25 n_{c}\right)$ in the intensity range $I \lambda_{0}^{2}=10^{19}-10^{20}$ $\mathrm{Wcm}^{-2} \mu \mathrm{m}^{2}$. Two different target thicknesses of $3.5 \lambda_{0}$ and $8 \lambda_{0}$ were considered. We have found that, in the case where a SPW is resonantly excited at the surface of the target, the maximum ion energy is increased up to a factor $\simeq 1.8$ with respect to the case of a flat target where no SPW is excited. The thinner target gives the higher ion energy. As previously observed ${ }^{18-21}$ grating targets thinner than $3.5 \lambda_{0}$ can be used to further increase the ion energy via enhancing electron recirculation, keeping in mind that the reducing of the target thickness is limited here by the needed modulation depth and experimental constraints in the elaboration of such grating. Optimisation of the depth of the modulation in order to improve the laser grating coupling ${ }^{38}$ may also be a way to increase the ion energy and will be explored in a future publication.

The variation of the maximum ion energy versus laser intensity has been also investigated and an optimal intensity range of $I \lambda_{0}^{2}=10^{19}-5 \times 10^{19} \mathrm{Wcm}^{-2} \mu \mathrm{m}^{2}$, which allows the efficient increase of the maximum ion energy via SPW excitation has been defined. Finally, comparing the case where the pulse has a Gaussian transverse profile with the case where the laser intensity is uniform on the target surface, we found that the ion cut off energy is increased by the same factor $(\simeq 1.8)$ in the two cases.

Thus, we may conclude that surface plasma wave excitation via intense laser-grating interaction is a promising tool to increase the energy of the accelerated particles for applications such as ion acceleration for hadron-therapy. $6,7,9,21,35$ This study opens new perspectives on the possibility of efficient coupling between laser and structured plasmas at relativistic intensity. The idea of using structured surfaces in order to take advantage of SPW excitation, already widely used at low intensity, ${ }^{36}$ can now be extended to laser-plasma interaction at high intensity, thanks to the plasma mirror technique, ${ }^{37}$ which allows to preserve the surface shape. To improve the ion energy, the perpendicular spot size of the laser should be increased or a super Gaussian for the focal spot could be used as suggested by comparison with the plane wave results. We also observed that during all the pulse duration in our simulation, the grating keeps its shape, so the possibility of taking longer pulse could be tested in the future.

\section{ACKNOWLEDGMENTS}

This work was performed using HPC resources from GENCI-CCRT (Grant 2011-2012-t2012056851). A.B. acknowledges support by French State Fonds managed by the ANR within the SimPLE projet (LABEX PALM-ANR-10-LABX-39).

${ }^{1}$ A. Maksimchuk, S. Gu, K. Flippo, D. Umstadter, and V. Yu Bychenkov, Phys. Rev. Lett. 84, 4108 (2000)

${ }^{2}$ E. L. Clark, K. Krushelnick, J. R. Davies, M. Zepf, M. Tatarakis, F. N. Beg, A. Machacek, P. A. Norreys, M. I. K. Santala, I. Watts, and A. E. Dangor, Phys. Rev. Lett. 84, 670 (2000).

${ }^{3}$ R. A. Snavely, M. H. Key, S. P. Hatchett, T. E. Cowan, M. Roth, T. W. Phillips, M. A. Stoyer, E. A. Henry, T. C. Sangster, M. S. Singh, S. C. Wilks, A. MacKinnon, A. Offenberger, D. M. Pennington, K. Yasuike, A. B. Langdon, B. F. Lasinski, J. Johnson, M. D. Perry, and E. M. Campbell, Phys. Rev. Lett. 85, 2945 (2000).

${ }^{4}$ S. C. Wilks, A. B. Langdon, T. E. Cowan, M. Roth, M. Singh, S. Hatchett, M. H. Key, D. Pennington, A. MacKinnon, and R. A. Snavely, Phys Plasmas 8, 542 (2001).

${ }^{5}$ P. Mora, Phys. Rev. Lett. 90, 185002 (2003); Phys. Rev. E 72, 056401 (2005).

${ }^{6}$ L. Robson, P. T. Simpson, R. J. Clarke, K. W. D. Ledingham, F. Lindau, O. Lundh, T. McCanny, P. Mora, D. Neely, C.-G. Wahlstrom, M. Zepf, and P. McKenna, Nature Phys. 3, 58 (2007).

${ }^{7}$ T. Bartal, M. E. Foord, C. Bellei, M. H. Key, K. A. Flippo, S. A. Gaillard, D. T. Offermann, P. K. Patel, L. C. Jarrot, D. P. Higginson, M. Roth, A. Otten, D. Kraus, R. B. Stephens, H. S. McLean, E. M. Giraldez, M. S. Wei, D. C. Gautier, and F. N. Beg, Nature Phys. 8, 139 (2012).

${ }^{8}$ S. Fritzler, V. Malka, G. Grillon, J. P. Rousseau, F. Burgy, E. Lefebvre, E. d'Humières, P. McKenna, and K. W. D. Ledingham, Appl. Phys. Lett. 83, 3039 (2003).

${ }^{9}$ V. Malka, S. Fritzler, E. Lefebvre, E. d'Humières, R. Ferrand, G. Grillon, C. Albaret, S. Meyroneinc, J. P. Chambaret, A. Antonetti, and D. Hulin, Med. Phys. 31, 1587 (2004).

${ }^{10}$ S. Atzeni, Phys. Plasmas 6, 3316-3326 (1999)

${ }^{11}$ J. Kupersztych, M. Raynaud, and C. Riconda, Phys. Plasmas 11, 1669-1673 (2004).

${ }^{12}$ M. Raynaud, J. Kupersztych, C. Riconda, J. C. Adam, and A. Heron, Phys. Plasmas 14, 092702 (2007).

${ }^{13}$ G. Hu, A. Lei, J. Wang, L. Huang, W. Wang, X. Wang, Y. Xu, B. Shen, J. Liu, W. Yu, R. Li, and Z. Xu, Phys. Plasmas 17, 083102 (2010).

${ }^{14}$ X. Lavocat-Dubuis and J.-P. Matte, Phys. Plasmas 17, 093105 (2010); Y. T. Li, X. H. Yuan, M. H. Xu, Z. Y. Zheng, Z. M. Sheng, M. Chen, Y. Y. Ma, W. X. Liang, Q. Z. Yu, Y. Zhang, F. Liu, Z. H. Wang, Z. Y. Wei, W. Zhao, Z. Jin, and J. Zhang, Phys. Rev. Lett. 96, 165003 (2006); W.-M. Wang, Z.-M. Sheng, and J. Zhang, Phys. Plasmas 15, 030702 (2008); G. Hu, A. lei, W. Wang, X. Wang, L. Huang, J. Wang, Y. Xu, J. Liu, W. yu, B. Shen, R. Li, and Z. Xu, ibid.. 17, 033109 (2010).

${ }^{15}$ O. Klimo, J. P. Psikal, J. Limpouch, J. Proska, F. Novotny, T. Ceccotti, V. Floquet, and S. Kawata, New J. Phys. 13, 053028 (2011).

${ }^{16}$ P. K. Kaw and J. B. McBride, Phys. Fluids 13, 1784-1790 (1970). 
${ }^{17}$ A. Bigongiari, M. Raynaud, C. Riconda, A. Héron, and A. Macchi, Phys. Plasmas 18, 102701 (2011).

${ }^{18}$ A. J. MacKinnon, Y. Sentoku, P. K. Patel, D. W. Price, S. Hatchett, M. H. Key, C. Andersen, R. A. Snavely, and R. R. Freeman, Phys. Rev. Lett. 88, 215006 (2002).

${ }^{19}$ Y. Sentoku, T. E. Cowan, A. Kemp, and H. Ruhl, Phys. Plasmas 10, 2009 (2003).

${ }^{20}$ E. d'Humières, E. Lefebvre, L. Gremillet, and V. Malka, Phys. Plasmas 12, 062704 (2005)

${ }^{21}$ J. Fuchs, P. Antici, E. d'Humières, E. Lefebvre, M. Borghesi, E. Brambrink, C. A. Cecchetti, M. Kaluza, V. Malka, M. Manclossi, S. Meyroneinc, P. Mora, J. Schreiber, T. Toncian, H. Pépin, and P. Auderbert, Nat. Phys. 2, 48 (2006).

${ }^{22}$ Developed at $\mathrm{CPhT}$, Ecole Polytechnique.

${ }^{23}$ A. Bigongiari, M. Raynaud, and C. Riconda, Phys. Rev. E 84, 015402(R) (2011).

${ }^{24}$ S. C. Wilks and W. L. Kruer, IEEE J. Quantum Electron. 33, 1954 (1997).

${ }^{25}$ P. J. Catto and R. M. More, Phys. Fluids 20, 704 (1977).

${ }^{26}$ W. L. Kruer and K. Estabrook, Phys. Fluids 28, 430 (1985).

${ }^{27}$ F. Brunel, Phys. Rev. Lett. 59, 52 (1987); Phys. Fluids 31, 2714 (1988).

${ }^{28}$ E. G. Gamaliy and R. Dragila, Phys. Rev. A 42, 929 (1990).

${ }^{29}$ K. Wharton, S. P. Hatchett, S. C. Wilks, M. H. Key, J. D. Moody, V. Yanovsky, A. A. Offenberger, B. A. Hammel, M. D. Perry, and C. Joshi, Phys. Rev. Lett. 81, 822 (1998).

${ }^{30}$ C. Beg, A. R. Bell, A. E. Dangor, C. N. Danson, A. P. Fews, M. E. Glinsky, B. A. Hammel, P. Lee, P. A. Norreys, and M. Tatarakis, Phys. Plasmas 4, 447 (1997).

${ }^{31}$ S. Gordienko and A. Pukov, Phys. Plasmas 12, 043109 (2005).

${ }^{32}$ A. Diaw and P. Mora, Phys. Rev. E 86, 026403 (2012).
${ }^{33}$ M. Borghesi, J. Fuchs, S. V. Bulanov, A. J. Mackinnon, P. K. Patel, and M. Roth, Fusion Sci. Technol. 49, 412 (2006).

${ }^{34}$ M. Borghesi, A. J. Mackinnon, D. H. Campbell, D. G. Hicks, S. Kar, P. K. Patel, D. Price, L. Romagnani, A. Schiavi, and O. Willi, Phys. Rev. Lett. 92, 055003 (2004).

${ }^{35}$ S. V. Bulanov and V. S. Khoroshkov, Plasma Phys. Rep. 28, 493 (2002); E. Fourkal, B. Shahine, M. Ding, J. S. Li, T. Tajima, and C. M. Ma, Med. Phys. 29, 2788 (2002); H. Schwoerer, S. Pfotenhauer, O. Jäckel, K.-U. Amthor, B. Liesfeld, W. Ziegler, R. Sauerbrey, K. W. D. Ledingham, and T. Esirkepov, Nature 439, 445 (2006).

${ }^{36} \mathrm{H}$. Reather, Surface Plasmons on Smooth and Rough Surfaces and on Gratings (Springer-Verlag, Berlin, 1988).

${ }^{37}$ G. Doumy, F. Quéré, O. Gobert, M. Perdrix, Ph. Martin, P. Audebert, J. C. Gauthier, J. P. Geindre, and T. Wittmann, Phys. Rev. E 69, 026402 (2004); J. Fuchs, P. Antici, E. d'Humières, E. Lefebvre, M. Borghesi, E. Brambrink, C. A. Cecchetti, T. Toncian, H. Pepin, and P. Audebert, J. Phys. IV 133, 1151 (2006); D. Neely, P. Foster, A. Robinson, F. Lindau, O. Lundh, A. Persson, C. G. Wahlstrom, and P. MacKenna, Appl. Phys. Lett. 89, 021502 (2006); T. Ceccotti, A. Levy, H. Popescu, F. Réau, P. d'Oliveira, P. Monot, J. P. Geindre, E. Lefebvre, and Ph. Martin, Phys. Rev. Lett. 99, 185002 (2007); C. Thaury, F. Quéré, J.-P. Geindre, A. Levy, T. Ceccotti, P. Monot, M. Bougeard, F. Réau, P. d'Oliveira, P. Audebert, R. Marjoribanks, and Ph. Martin, Nat. Phys. 3, 424-429 (2007); P. Antici, J. Fuchs, E. d'Humières, E. Lefebvre, M. Borghesi, E. Brambrink, C. A. Cecchetti, S. Gaillard, L. Romagnani, Y. Sentoku, T. Toncian, O. Willi, P. Audebert, and H. Pepin, Phys. Plasmas 14, 030701 (2007).

${ }^{38}$ A. Bigongiari, "High intensity laser-plasma grating interaction: Surface wave excitation and particle acceleration," Thesis (Ecole Polytechnique, 2012). 